\title{
Thomas Lemke
}

\section{Molekulare Medizin? \\ Anmerkungen zur Ausweitung und Redefinition des Konzepts der genetischen Krankheit}

\begin{abstract}
"Gesundheit wird zukünftig sowohl auf individueller wie gesellschaftlicher Ebene wesentlich an das Wissen um genetisch bedingte Dispositionen gebunden sein. Es ist abzusehen, dass diese genetische Lebenswelt für die Menschen genau so wahr werden wird, wie die hygienisch-bakteriologische Lebenswelt für uns wahr geworden ist." (Labisch 2002: 135f.; Hervorhebung im Original)
\end{abstract}

Im Verlauf des 20. Jahrhunderts hat sich die Rolle der Genetik in der Medizin fundamental verändert. ${ }^{1}$ In der ersten Jahrhunderthälfte spielten medizinische Fragen innerhalb der Genetik kaum eine Rolle. Die Humangenetik war eher ein Fachgebiet, das in die Zuständigkeit von Zoologen und Botanikern fiel, während die medizinischen Erfolge dieser Zeit vor allem auf die Verbesserung der Arbeits-, Wohn- und Ernährungsbedingungen sowie die Entwicklung von Impfstoffen und Antibiotika zurückgingen (Rushton 1994; Porter 1997). Selbst nach der Einführung der Genetik in die medizinischen Fakultäten im zweiten und dritten Viertel des letzten Jahrhunderts war deren klinische Relevanz auf vergleichsweise seltene so genannte Erbkrankheiten begrenzt. Dies hat sich inzwischen dramatisch geändert. Immer häufiger wird heute von einer „Ära der molekularen Medizin" (Ganten/Ruckpaul 2001: 3; vgl. Caskey 1995; Clark 1997; Williams/Hayward 2001) gesprochen und genetischen Erklärungsmodellen eine große Bedeutung für Diagnose, Prävention und Therapie vieler Zivilisationskrankheiten zugebilligt. So erklärt etwa die derzeitige Bundesministerin für Bildung und Forschung, Edelgard Bulmahn, dass die „Anwendung moderner molekularbiologischer Methoden und Erkenntnisse die tägliche medizinische Praxis revolutionieren " werde (Bulmahn 2003: 6). Angesichts dieser Zukunftsvisionen stellt sich die Frage, wie es zu dieser tief greifenden Veränderung in den letzten dreißig Jahren kommen konnte.

Der folgende Beitrag geht einer zentralen Voraussetzung für diesen einschneidenden Paradigmenwechsel nach: der Ausweitung und Neudefinition des

1 Dieser Artikel beruht auf Arbeiten im Rahmen eines Forschungsprojekts, das von der DFG unter dem Titel „Genetische Diagnostik in der Risikogesellschaft“ gefördert wird.

PROKLA. Zeitschrift für kritische Sozialwissenschaft, Heft 132, 33. Jg., 2003, Nr. 3, 471-492 
Konzepts der genetischen Krankheit. Nach einer Analyse der diskursiven Bedingungen für die aktuelle Neuorientierung der Medizin sollen abschließend einige soziale Implikationen dieser Transformation des Krankheitsverständnisses herausgestellt werden.

\section{Wissenschaftliche Erfolge, technische Innovationen, konzeptionelle Erfindungen: die langsame Etablierung des "genetischen Standpunkts"}

Für die wachsende Berücksichtigung molekularbiologischer Problemstellungen in der Medizin gibt es eine Reihe von historischen Entstehungsbedingungen. Scheinbar paradox liegt ein Grund für das medizinische Interesse an genetischen Krankheitserklärungen in dem Erfolg jenes Paradigmas, das dem molekulargenetischen Diskurs vorausging: das bakteriologische. Die wirksame Bekämpfung von Infektionskrankheiten durch Impfstoffe und Antibiotika Ende des 19. Jahrhunderts und in der ersten Hälfte des 20. Jahrhunderts veränderte das medizinische Wissen. Die Entdeckungen von Pasteur und Koch markierten nicht nur den Beginn der Labormedizin, sondern trugen auch zu einer weiteren Verwissenschaftlichung des Krankheitskonzepts bei (Latour 1988). Durch das bakteriologische Wissen nahmen Zahl und Schwere von Infektionskrankheiten insgesamt $a b$, so dass die allgemeine Lebenserwartung anstieg und die Säuglingssterblichkeit sank. Damit verlagerte sich das medizinische Interesse auf chronische Krankheiten wie Krebs, Diabetes, Herz-Kreislauferkrankungen etc. (Koch 1993: 5-7, Labisch 2002).

In der zweiten Hälfte des 20. Jahrhunderts rückte die Genetik durch die eindrucksvollen technischen und wissenschaftlichen Erfolge der 1950er bis 1980 er Jahre von einer vergleichsweise unbedeutenden Disziplin in den Mittelpunkt der Biowissenschaften. ${ }^{2}$ Dabei wuchs jedoch nicht nur ihre Bedeutung innerhalb der Biologie, sondern die Genetik machte selbst eine Transformation durch, die sie für medizinische Problemstellungen öffnete. Seit der Entdeckung der DNA-Technologie in den 1970er Jahren wurde aus einer Entdeckungsdisziplin, die der Grundlagenforschung verpflichtet war, eine Anwendungspraxis von potentiell großer medizinischer Bedeutung.

Hans-Jörg Rheinberger hat diesen Transformationsprozess aus wissenschaftsgeschichtlicher Perspektive detailliert beschrieben. Er wies darauf hin, dass die klassischen biophysikalischen, biochemischen und genetischen Techniken innerhalb der Molekularbiologie, die bis in die 1960er Jahre hinein zum Einsatz kamen, auf die Konstruktion einer experimentellen Umgebung zielten, die eine extrazelluläre Darstellung intrazellulärer Konfigurationen ermöglicht. Durch die Arbeit mit „Modellorganismen“ sollten zelluläre Strukturen er-

2 Zur Geschichte der medizinisch-genetischen Innovationen siehe Chadarevian/Kamminga (1998); Rheinberger (1998); Ganten/Ruckpaul (2001: 6-9). 
kannt und analysiert werden (Rheinberger 1996: 289f.). Rheinberger zufolge hat seit den 1970er Jahren die Art und Weise, in der molekulare Strukturen und Prozesse des Organismus der Manipulation zugänglich gemacht wurden, einen grundlegenden Wandel erfahren. Entscheidend war die Einführung rekombinanter DNA-Techniken, deren zentrale Werkzeuge wie Restriktionsenzyme, Transkriptasen etc. keine analytischen oder elektronischen Maschinen darstellen, sondern selbst biologische Makromoleküle sind. Daraus ergeben sich neue technologische Möglichkeiten. Indem das genetische Programm eines Organismus mit Hilfe seiner eigenen, modifizierten und unmodifizierten Komponenten verändert werden kann, verlässt die Molekularbiologie als molekulare Ingenieurwissenschaft das traditionelle Arbeitsparadigma und eröffnet zugleich eine neue Dimension medizinischen Handelns:

„Der Organismus selbst wird in ein Labor verwandelt. Von nun an zählt nicht mehr die extrazelluläre Repräsentation intrazellulärer Prozesse, d.h. das ,Verstehen' des Lebens, sondern vielmehr die intrazelluläre Repräsentation eines extrazellulären Projekts, d.h. die ,Umschreibung' des Lebens" (ebd.: 291; vgl. auch Rheinberger 1997)

Aber die Bedeutung der Genetik für die Medizin wuchs nicht nur aufgrund der technischen Innovationen und wissenschaftlichen Erfolge, zugleich wurde das bislang relativ starre Konzept genetischer Determination einer tiefgreifenden Revision unterzogen. Im lichte des wissenschaftlich-technologischen Fortschritts wird das Verhältnis von Anlage und Umwelt im Diskurs der Molekularbiologie seit dem Ende der 1960er Jahre neu begriffen. Die „Erbmasse“ erscheint zunehmend als offen gegenüber technischen Eingriffen und sozial gestaltbar. Der Bezug auf Gene bzw. molekulare Strukturen impliziert daher immer weniger Schicksalhaftigkeit und Unveränderlichkeit, im Gegenteil präsentiert sich die Genetik als ein privilegiertes Feld für soziale und medizinische Interventionen:

„Nach traditioneller Auffassung wurde „Erbanlage ${ }^{6}$ mit Schicksal und ,Umwelt' mit Freiheit verbunden; jetzt schienen die Rollen vertauscht zu sein. Die technischen Innovationen der molekularen Biologie ermutigten zu immer kühneren Schlußfolgerungen, die die Vorstellung stärkten, die Anlage sei leichter in den Griff zu bekommen als die Umwelt. [...] die Erfolge der Molekularbiologie lösten Vorstellungen aus, in denen die ,Erbanlage' auf neue Weise formbar erschien, und zwar bei weitem formbarer, als man das bei der ,Umwelt ${ }^{6}$ je für möglich gehalten hatte." (Keller 1995: 291f; vgl. Paul 1998a)

Diese Veränderungen blieben nicht ohne Folgen. Die 1970er waren eine entscheidende Dekade, in der sich molekurbiologische Techniken innerhalb der praktischen Medizin, vor allem im diagnostischen Bereich, langsam etablierten. In den USA und anderen Industriestaaten kamen gendiagnostische Verfahren in den ersten Screeningprogrammen zum Einsatz (Duster 1990; Wailoo 2001), und die Pränataldiagnostik wurde Teil der Schwangerenvorsorge (Rapp 2000). Trotzdem spielten genetische Fragestellungen in den 1970er Jahren innerhalb der Medizin weiterhin nur eine marginale Rolle, wie der Präsident der Amerikanischen Gesellschaft für Humangenetik, Barton Childs, 1976 in seiner 
Rede auf der Jahrestagung der Gesellschaft ernüchtert feststellt. ${ }^{3}$ Childs beklagt in dem Vortragstext, dass das genetische Wissen innerhalb der medizinischen Profession und Ausbildung kaum Aufmerksamkeit finde und die aus seiner Sicht notwendige institutionelle und konzeptionelle Reorganisation der Medizin ausbleibe. Er bezieht sich dabei auf eine Umfrage unter praktischen Ärzten zu genetischen Massenuntersuchungen, die zeigt, dass Mitte der 1970er Jahre weniger als die Hälfte der befragten Mediziner/innen genetische Krankheiten als ein ernstes Problem für ihre klinische Arbeit ansehen (Rosenstock et al. 1975). Childs untersucht zahlreiche Restriktionen und die traditionellen Strukturen in der ärztlichen Praxis, aber auch in der wissenschaftlichen Forschung und Vermittlung medizinischen Wissens, welche die von ihm als unverzichtbar erachtete Berücksichtigung genetischer Faktoren innerhalb der Medizin verhindern. Genauer geht er auf vier Gründe ein, um zu erläutern, warum Mediziner/innen (noch) nicht in genetischen Begriffen denken: Erstens konzentriere sich die Arbeit von Ärzten traditionell mehr auf Individuen als auf Familien. Zweitens gehe es in der bisherigen medizinischen Praxis eher um einzelne Krankheitsfälle denn um allgemeine Prävention und Gesundheitsaufklärung. Drittens habe die Spezialisierung in der Medizin auch die Genetik getroffen, da die genetische Beratung stark von der Forschung abhängig sei, die nur in spezialisierten Krankenhäusern durchgeführt werde. Schließlich konzentrierten sich die Ärzte auf die akute Behandlung von Patienten, während umfassendere gesundheitspolitische Probleme innerhalb der klinischen Arbeit ausgeblendet blieben. Daher werde jemand mit einer genetischen Krankheit allein als unmittelbar Betroffener, nicht aber im Hinblick auf eine mögliche Weitergabe dieser Krankheit an die Nachkommen betrachtet (Childs 1977: 7; vgl. Yoxen 1984: 50-52; Koch 1993). Der von Childs eingeforderte "genetische Standpunkt" (Childs 1977: 8), der bei praktischen Ärzten und den Studenten der Medizin zu verankern und zu kultivieren sei, sollte es demgegenüber erlauben, eine bessere Erklärung von Unterschieden und Ähnlichkeiten bei Personen, die an derselben Krankheit leiden, zu liefern und den ärztlichen Blick über das einzelne Individuum hinaus auf dessen Familie und sogar die gesamte Menschheit erweitern (ebd., 8f.).

Zwischen der äußerst begrenzten Aufnahme genetischer Erklärungsprinzipien und technologischer Anwendungen in der medizinischen Praxis der 1970er Jahre und der heutigen „Ära der molekularen Medizin“ liegen offenbar wissenschaftshistorische Welten. In den letzten 25 Jahren hielt der „genetische Standpunkt" in einer Weise Einzug in die Medizin, dass der inzwischen emeritierte Childs 1999 in einem Buch mit dem Titel „Genetic Medicine. A Logic

3 Die Rede wurde tatsächlich niemals gehalten, da die Amerikanische Gesellschaft für Humangenetik 1976 nicht tagte, sondern am Internationalen Kongress der Humangenetik in Mexico City teilnahm (Childs 1977: 1). 
of Disease ${ }^{65}$ von einem Zusammenwachsen von Genetik und Medizin und einer neuen Logik der Krankheitserklärung spricht:

„Spontaneously and gradually, a synthesis of genetic and medical ideas [...] is now beginning. It is a wedding of equals, and its offspring affords us a new logic, a new way of examining old questions of the nature of disease and its causes" $(1999: x){ }^{4}$

In den vergangenen Jahrzehnten ist es der Genetilk gelungen, sich als medizinische Grundlagenwissenschaft zu etablieren, die nicht nur für einzelne und sehr seltene vererbte Krankheiten, sondern für die Genese und Manifestation von Krankheiten überhaupt zuständig sein soll. Ein Beispiel für diesen Entwicklungstrend ist das mehrbändige „Handbuch der Molekularen Medizin“, das von monogen bedingten Erbkrankheiten bis hin zu Tumorleiden und Herz-Kreislauf-Beschwerden eine große Palette von Krankheiten umfasst (Ganten/Ruckpaul 1997ff.). Den Herausgebern zufolge zeichnet sich mit der molekularen Medizin ein epochaler Einschnitt in der medizinischen Praxis ab:

„Mit der Erschließung der genomischen Ebene als Angriffspunkt für Diagnostik, therapeutische Interventionen und Prävention stehen wir am Beginn einer neuen Ära der Molekularen Medizin, deren wesentliches Kennzeichen die Anwendung von gentechnischen Methoden für die Erkennung, Behandlung und Vorbeugung von Krankheiten auf molekularer Ebene ist." (Ganten/Ruckpaul 2001: 3; vgl. Kaplan/Junien 2000)

Die "Gen-Medizin“ (Raem et al. 2001) eröffnet demzufolge eine genauere Analyse der Pathogenese durch eine neue Taxonomie von Krankheiten, da - so der formulierte Anspruch - bislang nur phänotypisch beschreibbare Krankheitszustände durch genotypische Veränderungen erklärt und verstanden werden könnten (Ganten/Ruckpaul 2001: 8). Das Ziel dieser Neuorientierung innerhalb der Medizin sei es, ein neues Modell der Verifikation von Krankheitsursachen zu etablieren, das Krankheiten nicht mehr nach ihrem klinischen Erscheinungsbild beurteilt, sondern auf deren molekulargenetische „Ursachen“ zurückführt. Daran knüpft sich die Hoffnung, die Unterteilung nach genetischen Kriterien werde es ermöglichen, die vielfältigen Variationen dieser Krankheiten ebenso zu erklären wie die offensichtlichen Differenzen im Krankheitsbild, dem klinischen Verlauf und der Reaktion auf Therapien. In dieser Perspektive soll die Molekulargenetik die Werkzeuge bereitstellen, um Krankheiten nach den ihnen zugrunde liegenden Mechanismen zu definieren. Dieser epistemologische Einschnitt wird auch in einem anderen Handbuch zur Gen-Medizin herausgestellt. Der molekulare Ansatz werde

„in vielen Bereichen zu einem ganz neuen Verständnis der Medizin führen, das in absehbarer Zeit auch Relevanz für den Kliniker und den Patienten erhalten wird. Die Analyse der Krankheitssymptomatik wird immer stärker durch eine genetisch-kausale Seite ergänzt werden" (Maurer/Lehrach 2001: XIII; Bell 1998).

4 Daher wird immer häufiger gefordert, das genetisch-diagnostische Wissen in die ärztliche Grundversorgung zu integrieren: „The likely increases in availability of DNA based tests and demand by patients for genetic information and advice mean that primary care practitioners will need to become genetically literate" (Emery 2001: 1027; Caskey 1995: 155; Harris/Haris 1995). 
Entscheidend für die wachsende Relevanz molekularbiologischer Problemstellungen für die medizinische Forschung und die klinische Praxis ist die Ausdehnung und Reformulierung des Begriffs der genetischen Krankheit, die es erlaubt, den traditionellen medizinischen Fokus auf das Individuum beizubehalten und diesen zugleich - wie von Childs bereits Mitte der 1970er Jahre gefordert - mit allgemeinen gesundheitspolitischen Überlegungen und Optionen zur Krankheitsprävention zu verknüpfen.

\section{Symptomatologie und Verifikation}

Seit Anfang der 1990er Jahre werden für immer mehr Krankheiten genetische Faktoren verantwortlich gemacht. Diesem Krankheitskonzept liegt die Annahme zugrunde, dass ein oder mehrere "defekte ${ }^{66}$ Gene zum Verlust oder der Beeinträchtigung einer Stoffwechselfunktion fïhren, die schließlich für eine Krankheit kausal verantwortlich ist oder den Organismus für bestimmte Krankheiten stärker disponiert. Die Entwicklung hin zu einer "Genetisierung der Medizin" (Lippman 1991; Höhn 1997; Schmidtke 1998) lässt sich anhand von zwei wichtigen Indikatoren illustrieren. Seit dreißig Jahren werden Krankheiten, von deren Erblichkeit ausgegangen wird, durch den sog. McKusickKatalog erfasst. Dieser Katalog, der Mendelian Inheritance in Man (MIM) ${ }^{5}$ ist die entscheidende medizinische Standarddatenbank, die Auskunft gibt über die Anzahl erblicher Erkrankungen. Sie wird jede Woche auf den neuesten Stand gebracht. Die Zahl der im McKusick-Katalog verzeichneten Krankheitsbilder, für die genetische Ursachen bekannt sind, betrug im Jahr 1992 5.000, stieg auf 10.000 Ende 1998 und liegt heute bei über 14.000 Einträgen (24. April 2003: 14.372; http://www.ncbi.nlm.nih.gov/Omim/Stats/ mimstats.html). ${ }^{6}$

Ebenso beeindruckend ist der Aufstieg des Konzepts des genetischen Risikos (genetic risk) in der Medizin. Eine Recherche in der Literaturdatenbank MEDLINE, in der alle wichtigen medizinischen Fachzeitschriften indexiert sind, dokumentiert einen fast unglaublichen Anstieg von Artikeln, die sich in den letzten dreißig Jahren mit genetischen Risiken beschäftigten.? Während im Zeitraum zwischen 1967 und 1971 nur vier Artikel aufgeführt sind, die genetic

5 Die Internet-Version OMIM (Online Mendelian Inheritance in Man) besitzt folgende Adresse: http:/ www.ncbi.nlm.nih.gov/Omim.

6 Diese Entwicklung bleibt nicht ohne Folgen für die medizinische Diagnostik. Inzwischen sind nicht nur für wenige Erbkrankheiten, sondern auch für einige der häufig auftretenden Zivilisationskrankheiten wie Krebs, Diabetes und Alzheimer genetische Nachweisverfahren verfügbar. In der Bundesrepublik Deutschland, Österreich und der Schweiz stieg in den letzten Jahren die Zahl molekulargenetischer Tests stark an. Zur Zeit können mehr als 400 genetische Dispositionen mittels genetischer Anwendungen diagnostiziert werden. Siehe das Angebot von molekulargenetischen Leistungen des Bundesverbandes Medizinische Genetik: http://www.bvmedgen.de/qs/aktumole.html.

7 MEDLINE ist zu erreichen über folgende Internetadresse: http://www.ncbi.nlm.nih.gov/ entrez/query.fcgi. 
risk im Titel und/oder Abstract enthalten, waren es zehn Jahre später schon 67 (1977-1981); in den fünf Jahren zwischen 1987 und 1991 wuchs die Zahl auf 211 an, um dann nochmals zu steigen: auf 1082 (1997-2001). Mit anderen Worten: In den letzten 20 Jahren ist die Zahl der Beiträge in medizinischen Zeitschriften, die sich auf die Analyse genetischer Risiken konzentrierten, um den Faktor 15 angestiegen. ${ }^{8}$

Die angeführten Indikatoren dokumentieren, dass innerhalb der medizinischen Forschung offenbar immer mehr Krankheitsursachen auf genetische Mechanismen zurückgeführt werden. Dabei sind zwei Entwicklungstrends zu beobachten. Zum einen findet eine Ausweitung des Begriffs der genetischen Krankheit statt. Genetische Faktoren sollen nicht nur für "monogenetische" Krankheiten (die so genannten Erbkrankheiten), sondern auch multifaktorielle Erkrankungen wie Krebs, Alzheimer, Diabetes und viele andere Bevölkerungskrankheiten verantwortlich sein.? Zum anderen kommt es zu einer Neudefinition des Begriff der genetischen Krankheit. Die quantitative Ausdehnung des Begriffs der genetischen Krankheit wird durch eine qualitative Verschiebung ergänzt. Der Wissenschaftshistoriker Edward Yoxen hat vor zwanzig Jahren darauf hingewiesen, dass die Relation zwischen der Humangenetik und den medizinischen Wissenschaften dabei ist, sich entscheidend zu ändern. Er diagnostizierte einen Prozess der Redefinition, der dazu führte, Krankheiten immer mehr als genetische Normabweichungen zu identifizieren. Yoxen zufolge bereitete erst die Ausweitung der Vorstellung von „genetischen Krankheiten ${ }^{6}$ und die Begriffsverschiebung den Boden für die wachsende gesellschaftliche und medizinische Bedeutung der Molekulargenetik (Yoxen 1984; vgl. Koch 1993). In der Folgezeit verwandelte sich der Begriff der genetischen Krankheit in eine "Mammutkategorie“ (Keller 1995: 296), die unterschiedliche Kausalbeziehungen und Krankheitskonzepte miteinander vermengt:

„(1) abnormalities having a genetic cause, but not leading to any physiological or behavioral, disorder, which we may call genetic disorders; and (2) genetic disorders that are apprehended as diseases and (3) disorders that are neither genetic nor diseases, even though they may be labeled as such by some people." (Yoxen 1984: 49)

Auf diese Weise hat sich ein produktiver Zirkel etabliert, der von genetischen Anomalien auf Krankheiten schließt und Krankheiten auf genetische Anomalien zurückführt.

8 Dieser Anstieg ist nicht auf das Stichwort genetic risk beschränkt; ähnliche Zahlen lassen sich für denselben Zeitraum auch für genetic susceptibility (von 5 Nachweisen zwischen 1967 und 1971 auf 1124 zwischen 1997 und 2001) und für genetic predisposition (Anstieg von 9 auf 1254 Nachweise im selben Zeitraum) dokumentieren.

9 Außerhalb des medizinischen Bereichs gehen einige Verhaltensgenetiker sogar so weit, genetische Faktoren auch für „soziale Krankheiten“ wie Arbeitslosigkeit, Alkoholismus, Kriminalität etc. verantwortlich zu machen. Für einen Überblick: Carson/Rothstein (1999); Wasserman/Wachbroit (2001). 
Die Redefinition von Krankheiten als genetische Erkrankungen und die Suche nach den ihnen „zugrunde liegenden" genetischen Mechanismen führt also nicht nur zu einer Ausweitung der Zahl genetischer Krankheiten; wenn innerhalb des Konzepts einer „molekularen Medizin" potentiell jede Krankheit auf eine genetische Veränderung zurückgeführt wird, ist fraglich, worin der Beitrag genetischer Faktoren genau besteht bzw. wie genetische Krankheitsursachen von nicht-genetischen abzugrenzen sind ${ }^{10}$ Mit der zunehmenden Etablierung einer Gen-Medizin verbinden sich zwei wichtige semantische Verschiebungen. Zum einen ist die Kategorie "genetisch bedingt "so weit ausgedehnt worden, dass sie heute keineswegs nur Verhaltensauffälligkeiten und Krankheitsformen umfasst, die durch die Keimbahn weitergeben werden, also "genetisch bedingt ${ }^{46}$ sind im herkömmlichen Sinn des Wortes (Keller 2001: 201, FN. 37). Der Begriff der genetischen Krankheit bezieht sich heute nicht nur auf vererbte Genveränderungen, sondern umfasst auch so genannte somatische Mutationen, d.h. Mutationen, die spontan auftreten und erworben werden. Insofern gilt: „Auch nicht-erbliche Erkrankungen können genetisch bedingt sein ${ }^{65}$ (Schmidtke 1998). Zum anderen erlaubt es der ,genetische Standpunkt $t^{66}$, den Krankheitsbegriff auf Zustände und Normvariationen auszudehnen, die bislang nicht als „krank ${ }^{6}$ angesehen wurden. Wenn genetische Abweichungen die Grundlage für die Bestimmung von Krankheiten liefern, so können auch genetische Anlagen, Dispositionen, Risiken etc. in den medizinischen Blick genommen werden, die sich (noch) nicht klinisch manifestiert haben. Damit ist das Modell einer präventiven oder prädiktiven Medizin skizziert, die sich von einer konkret beschreibbaren oder empirisch feststellbaren Krankheitssymptomatik abzukoppeln vermag. Auf der Ebene des Genotyps muss sich eine Krankheit nicht notwendig in einem bestimmbaren Krankheitsbild „zeigen“; im Gegenteil ist es das Ziel der molekularen Medizin, das Auftreten der konkreten Symptomatiken zu verhindern.

Es ist also eine doppelte epistemologische Grundlage für die Ausweitung des Begriffs der "genetischen Krankheit" zu konstatieren: Die qualitative Redefinition von Krankheiten unter genetischen Vorzeichen wird ergänzt durch deren kognitive Virtualisierung, durch die Entkoppelung der Krankheit von ,ihren" Symptomen, ja dieser Prozess der analytischen Abtrennung und Aufspaltung gilt sogar als Ausweis der Wissenschaftlichkeit. Die ,unklaren“ Symptomatiken auf der Ebene des Phänotyps verstellen - so die zugrunde liegende An-

10 Zum Problem der Ausblendung nicht-genetischer Faktoren durch die Wahl der molekularen Erklärungsebene siehe Janich (2001: 88): „Wenn entsprechend der erfolgreiche Rückgang auf die molekulare Beschreibung des menschlichen Genoms umgekehrt wird und der Mensch nur noch als Realisierung des genetischen Bauplans interpretiert wird, ist selbstverständlich alles verloren, was nicht genetisch bedingt ist. Insbesondere wird, erkenntnistheoretisch gesehen, die Offenheit für die Frage verloren, wie das genetisch Bedingte vom nicht genetisch Bedingten methodisch sauber zu unterscheiden ist." 
nahme - den Blick auf die "wirklichen" genetischen Mechanismen, deren Verständnis erst eine genaue Differenzierung und Systematisierung der Krankheitsursachen erlaubt. Auf diese Weise können jedoch auch Menschen als „krank ${ }^{66}$ betrachtet und behandelt werden, die völlig symptomfrei sind, da ihre "Krankheit" allein auf molekulargenetischer Ebene zu diagnostizieren ist. Damit stellt sich die Frage, mit welchem Krankheitsbegriff die Medizinische Genetik operiert.

\section{Was ist eine genetische Krankheit?}

Was macht eine bestimmte Anomalie oder beobachtbare Funktionsstörung zu einer genetischen Krankheit? Diese Frage ist alles andere als trivial, denn selbst in Lehrbüchern der Medizinischen Genetik finden sich unterschiedliche, häufig inkompatible Krankheitsbegriffe. ${ }^{11}$ In medizinhistorischer Perspektive ist es wichtig zu beachten, dass einige Dysfunktionen als Krankheiten angesehen wurden, bevor sie als genetisch verursacht betracht wurden (ein Beispiel dafür ist die Sichelzellenanämie). Umgekehrt wurden bestimmte Abnormalitäten als genetisch klassifiziert, bevor sie als Krankheiten angesehen wurden (dies betrifft Formen von Hämoglobin, die nicht unbedingt medizinisch relevant sind) (Yoxen 1984: 48f.).

Kelly C. Smith diskutiert in einem instruktiven Artikel die Frage, in welchem Sinn Krankheiten als genetisch verursacht oder „bedingt" betrachtet werden können (Smith 2001). Er kritisiert eine Reihe von Interpretationsmodellen, die er aus verschiedenen Gründen für unzureichend oder irreführend hält. ${ }^{12}$ Ein erster prominenter Definitionsvorschlag, der immer wieder in der medizinischen Literatur auftaucht, lautet, dass eine Krankheit immer dann als genetisch zu bezeichnen ist, wenn genetische Faktoren bei der Krankheitsentstehung eine kausale Rolle spielen. Wie Smith zeigt, scheint diese Annahme zunächst plausibel, bringt aber letztlich keinen Erkenntnisgewinn, da sie ebenso wahr wie inhaltsleer ist. In diesem Sinn kann von jeder Krankheit behauptet werden, dass ihr die Qualität ,genetisch“ zukommt und zwar schon deshalb, weil Gene für Proteine kodieren, welche für das Leben eines Organismus unverzichtbar sind. Diesem umfassenden Definitionsvorschlag zufolge könnte selbst eine Bleivergiftung als eine "genetische Krankheit" aufgefasst werden, da Menschen sehr unterschiedliche körpereigene und genetisch regulierte Mechanismen besitzen, um auf toxische Substanzen zu reagieren.

Der zweite von Smith vorgestellte Ansatz wählt als Auswahlkriterium nicht den einfachen Beitrag von Genen an der Entstehung einer Krankheit, sondern zielt auf das Moment der Kontrolle und Steuerbarkeit: „The manipulability

11 Vgl. die Beispiele, die Hoedemaekers anführt (1998: 92f.).

12 Die folgenden Überlegungen konzentrieren sich auf zwei Modelle, die Smith in seinem Beitrag ausführlicher diskutiert. 
criterion says that a disease is genetic if and only if it is best controlled (prevented) through manipulation of the genes" (Smith 2001: 22). Aber auch dieser Definitionsversuch wirft Probleme auf. Es stellt sich nämlich die Frage, was „am besten“ heißen soll. Eine konkrete Krankheit kann auf sehr unterschiedliche Weise behandelt werden. Smith führt als Beispiel Mukoviszidose ${ }^{13}$ an. Die Krankheit könne möglicherweise einmal mittels gentechnologischer Interventionen oder durch den Einsatz einer Maschine zum Inhalieren ,geheilt ${ }^{\text {s }}$ werden. Was „am besten " jeweils meint, ist keine Funktion technischwissenschaftlichen Fortschritts, sondern abhängig von sozialen Auseinandersetzungen, politischen Interessen, finanziellen Restriktionen, ökonomischen Profitmotiven etc.

Ähnlich wie Smith kommt auch Hoedemaekers nach einer ausführlichen Diskussion vorherrschender Krankheitskonzepte zu dem Schluss, dass es nur in sehr wenigen Fällen angemessen und hilfreich ist, von „genetischen Krankheiten" zu sprechen. Er empfiehlt den Begriff für jene seltenen monogenetischen Krankheiten zu reservieren, bei denen die genetische Veränderung sich weitgehend unabhängig von Umweltfaktoren in einer bestimmten Symptomatik manifestiert, in allen anderen Fällen aber sei es besser, von „genetischen Risiken" zu sprechen. ${ }^{14}$ Hoedemaekers möchte den Begriff der genetischen Krankheit also auf solche genetischen Anomalien begrenzt sehen, die einem streng deterministischen Modell der Krankheitsexpression folgen, wohingegen eine lediglich erhöhte Erkrankungswahrscheinlichkeit durch den Risikobegriff bezeichnet werden soll (Hoedemaekers 1998: 79-99; vgl. auch Hoedemaekers/ten Have 1999). . $^{15}$

Das immer wieder angeführte Musterbeispiel für die Möglichkeiten einer genetischen Medizin ist die erfolgreiche Behandlung von PKU, eine Stoffwechselstörung, bei der ein Enzymdefekt verhindert, dass die Aminosäure Phenylalanin in Tyrosin umgebaut werden kann. Allerdings zeigt gerade eine genaue Analyse der Geschichte der PKU-Behandlung die Grenzen eines Ansatzes auf, der sich allein auf molekulargenetische Mechanismen und Interventionsmög-

13 Mukoviszidose (oder Cystrische Fibrose) ist eine erbliche Stoffwechselstörung, die zu Komplikationen bei Atnuung und Verdauung führt.

14 Es gibt jedoch eine Reihe von Hinweisen darauf, dass selbst bei monogenetischen Krankheiten wie Mukoviszidose oder Huntington Umweltbedingungen eine wichtige Rolle spielen (Kerr 2000; Kaplan/Junien 2000: 1171; Van den Boer-van den Berg/Maat-Kievit 2001; Kaprio 2001).

15 Ein ähnliches Argument findet sich bei Temple 2001. Dieser Vorschlag - also den Begriff der genetischen Krankheit durch den des genetischen Risikos tendenziell zu ersetzen - vermag jedoch nicht zu überzeugen, da er das Problem nur verlagert, ohne es zu lösen. Alles hängt dann von der präzisen Definition des genetischen Risikos und der konkreten Abgrenzung von "Krankheit" zu (genetischen) „Krankheitsrisiken" ab. Zu beobachten ist jedoch, dass innerhalb der Genomforschung und in der medizinischen Genetik der Risikobegriff mindestens ebenso unbestimmt verwendet wird wie der Begriff der genetischen. Krankheit (vgl. dazu ausführlicher Lemke 2002). 
lichkeiten konzentriert (vgl. dazu ausführlich Paul 1998b). Zwar ist es möglich, PKU als eine genetische Krankheit zu bezeichnen, aber die Stoffwechselstörung kann wirksam durch eine bestimmte Diät verhindert werden. Zu sagen, dass eine Krankheit genetisch bedingt sei, heißt also nicht automatisch, dass es geboten oder sinnvoll ist, nach genetischen Faktoren für Prävention und Therapie zu suchen. Eher ist eine pragmatische Herangehensweise erforderlich, die weniger auf die vollständige Analyse der Krankheitsursachen denn auf die selektive Unterbrechung bzw. die Steuerung der Symptomatik zielt. Strategien der Krankheitsprävention gründen sich daher nicht notwendig in einer umfassenden und erschöpfenden Analyse der Pathogenese, sondern in der Bestimmung von Eingriffspunkten, die das Auftreten von Krankheitssymptomen wirksam unterbinden (im Extremfall kann auch ein falsches Krankheitsverständnis den Ausbruch einer Krankheit verhindern). In diesem Sinn kann eine Krankheit auf mehr als eine Weise vermieden werden - selbst wenn es sich um traditionelle "Erbkrankheiten“ wie PKU handeln mag: „Phenylketonuria is a classic case: the clinical disease can be avoided either by not having the genetic mutation or by elimination phenylalanine from the diet" (Willett 2002: 695).

Selbst wenn also die Molekulargenetik den hochgesteckten Ansprüchen genügen sollte und wichtige neue Kenntnisse über Krankheitsverläufe und -ursachen liefert, bliebe doch dahingestellt, ob der "genetische Standpunkt" für die klinische Forschung und die Entwicklung medizinischer Therapien von der erwartet großen Bedeutung ist. Aus der Diagnose einer genetischen Krankheit (was auch immer dies nach der obigen Diskussion der unterschiedlichen Analysemodelle konkret heißen mag), folgt keineswegs, dass diese auch genetisch behandelt werden muss, denn im Rahmen der klinischen Arbeit geht es nicht darum, die „Ursache für Krankheiten zu finden, sondern deren „Achillesferse $^{\text {" }}$ (Rees 2002; Smith 2001: 19f.). Aber die Definition und Diagnose von genetischen Krankheiten wirft noch ein grundsätzlicheres Problem auf: die Frage danach, welche genetischen Variationen als krankhaft und welche als medizinisch irrelevant angesehen werden.

\section{Variation und Selektion}

Das Versprechen einer molekularen Medizin ist es, Krankheiten einer wissenschaftlichen (nämlich: genetischen) Klassifikation unterziehen zu können. Die detaillierte Beschreibung molekularer Krankheitsursachen - so die erkenntnisleitende Prämisse - werde es der medizinischen Forschung erlauben, bessere und präzisere ( $\mathrm{da}$ am Gentyp orientierte) präventive Maßnahmen und therapeutische Techniken zu entwickeln.

Diese Präsentation und Vision einer molekularen Medizin vermischt jedoch zwei prinzipiell zu trennende analytische Ebenen. Auf der Ebene des Genoms 
gibt es keine Krankheiten, naturwissenschaftlich feststellbar sind nur individuelle Variationen. Selbst wenn in molekularbiologischen Lehrbüchern immer wieder über „Krankheitsgene“ geschrieben wird (z.B. Strachan/Read 1996: 453), so ist dieser Ausdruck doch irreführend und letztlich falsch, denn krank sein können nur Menschen oder andere Lebewesen. Von „kranken" Genen lässt sich wissenschaftich nicht reden. Die Charakterisierung von bestimmten Zuständen als "krank" oder "abnorm" ist eine soziale und moralische Definition, die „Handlungsbedarf ${ }^{\text {(s }}$ signalisiert und Interventionen initiieren und legitimieren soll. Die Identifikation von Krankheiten ist daher kein deskriptivwissenschaftlicher Vorgang, sondern impliziert immer ein normatives Urteil über die Veränderungsbedürftigkeit von Zuständen。 ${ }^{16}$ Die Bestimmung von Krankheiten basiert auf dem Vergleich mit Merkmalen oder Bedingungen, die als normal, ideal oder gesund angesehen werden. Mit anderen Worten: Entgegen dem eigenen Anspruch kann es die Molekulargenetik durch die Kennzeichnung genetischer Krankheiten nicht vermeiden, fundamentale Werturteile in Bezug auf Gesundheit und Krankheit zu fällen. Zwar kann sie

„das menschliche Genom detailliert bis in die einzelnen Bestandteile beschreiben. Bei dieser Vorgehensweise entdeckt man freilich allenfalls Vielfalt, niemals jedoch Krankheit. Auch die zahlreichen Versuche, über Modelle des Schadens, der Entgleisung, der Normwidrigkeit, des Ungleichgewichts, der mangelnden Harmonie, der speziestypischen Funktion oder ähnlichem nach einem umfassenden Kriterium für Krankheit zu suchen, haben an den grundsätzlichen Schwierigkeiten nichts geändert. Denn in diesen Fällen hat man längst mit den aufgezählten Modellen der Abweichung ein Kriterium herangezogen, das nicht so ohne weiteres einer Beschreibung der Natur zu entnehmen ist." (Wiesing 1998: 96; Koch 1999: 191f.)

In dieser Hinsicht gibt es keine wissenschaftich-neutrale Beschreibung von Krankheiten, sondern die Definition von Krankheiten ist selbst ein wertbezogener Prozess, der Entscheidungshandlungen impliziert. Ein Krankheitsbegriff, der diese normative Dimension ausblendet, läuft Gefahr, fundamentale und unumgängliche moralische Entscheidungen zu maskieren. ${ }^{17}$ Das molekulargenetische Wissen allein kann keinen Beitrag zu der Frage leisten, ob ein Zustand behandelt werden soll oder nicht. Es ist vielmehr mit angebbaren Gründen zu urteilen, welche „Abweichungen" als pathologisch charakterisiert und vermieden bzw. behandelt werden sollen. Es muss notwendigerweise entschieden werden, wo die Grenze zwischen normaler Variation und pathologi-

16 Aus Platzgründen bleibt hier die weitergehende Frage ausgeklammert, ob sich Krankheit überhaupt als ein rein naturwissenschaftliches Phänomen unabhängig von kulturellen Faktoren begreifen lässt, ob also die Spaltung von subjektiver Krankheitserfahrung und objektivem Krankheitsbefund, illness und disease, prinzipiell haltbar ist. $\mathrm{Zu}$ einer grundlegenden Kritik an dem biomedizinisch-naturwissenschaftlichen Zugang zum Problem der Krankheit vgl. Morris (2000); Fadiman (2000).

17 In diesem Sinn „ist der Vorgang, etwas als Krankheit zu bezeichnen, im Grunde ein moralisches Unternehmen mit moralischen Konsequenzen“ (Freidson 1979: 283, zit. nach Wiesing 1998: 96). Vgl. zu diesem Problemkomplex Hoedemaekers (1998: 81-88) und die Beiträge in dem Sammelband von Brandt/Rozin (1997). 
scher Mutation verläuft und welche Abweichung von der Norm als krankheitsrelevant anzusehen ist. In der gentechnischen Perspektive verliert die Krankheit ihren scheinbar „selbst-verständlichen " und „natürlichen" Status, sie wird zu etwas Problematischem, das definitorischer Festlegungen und gesellschaftlicher Diskussionen bedarf. Der bekannte Molekularbiologe French Anderson hat dieses Problem folgendermaßen formuliert:

„Was ist eine Krankheit? Wir können alle erkennen, was eine schwere Krankheit ist, weil sie schweres Leiden und vorzeitigen Tod verursacht. Wenn man aber diese Kategorie verläßt und auf geringfiigige Krankheiten kommt, was wäre dann geringfügig? Was ist überhaupt eine Krankheit? Was ist normal?" (Anderson zit. nach Lau/Keller 2001: 85).

Urban Wiesing weist zu Recht darauf hin, dass ein zentrales Kriterium für das Vorliegen einer Krankheit in dem subjektiven schlechten Befinden und dem individuellen Gefühl der Hilfsbedürftigkeit liegt. Auch wenn diese „Selbsteinschätzung ${ }^{6}$ nur mit einigen Qualifizierungen und Beschränkungen herangezogen werden kann, bleibt sie unverzichtbar: „Andernfalls würde man einen Menschen als ,krank ${ }^{6}$ und seinen Zustand als veränderungsbedürftig bezeichnen, der das selbst gar nicht so sieht und sich gar nicht verändern lassen will ${ }^{\text {is }}$ (Wiesing 1998: 97). Das strukturelle Problem der genetischen Medizin könnte möglicherweise darin bestehen, dass diese - entgegen des immer wieder ins Feld geführten Anspruchs der Patientenautonomie - gerade nicht auf die Selbsteinschätzung der Betroffenen rekurriert, sondern sich an einem scheinbar objektiven Krankheitsbegriff orientiert, der allein auf molekulare Vorgänge abstellt:

„Nicht die Selbstinterpretation eines Menschen wird zum ausschlaggebenden Moment für ärztliches Handeln, sondern eine bestimmte Genkonstellation. Und da Selbstinterpretation und Genkonstellation keineswegs immer korrelieren, liegt hier eine Gefahr." (Wiesing 1998: 97)

Auch der Wissenschaftsphilosoph Philip Kitcher hat auf diese "verborgenen“ normativen Grundlagen molekulargenetischer Erklärungsversuche aufmerksam gemacht. Kitcher zeigt, dass die Annahme, es gebe Gene "für" menschliche Krankheiten, Verhaltensmerkmale und Eigenschaften immer „die Vorstellung eines Standardhintergrunds und einer Standardumgebung" bzw. einer "Norm ${ }^{66}$ (1998: 275) voraussetzt. Diese Standardumgebungen können sozialer oder biologischer Art sein, sie sind dafür verantwortlich, ob und in welcher Weise eine bestimmte "genetische Abweichung" tatsächlich zur Veränderung des betreffenden Merkmals führt (vgl. dazu auch Conrad 1999: 234). Kitcher unterstreicht damit nicht nur die Notwendigkeit, normative Erwägungen in genetische Erklärungen einzubeziehen, er verweist auch auf ein weiteres Problem des Ansatzes einer "molekularen Medizin“: die tendenzielle Vernachlässigung oder gar systematische Ausblendung des Beitrags von Umweltfaktoren an der Entstehung von Krankheiten.

Die analytische Konzentration auf molekulare Krankheitsursachen macht unkenntlich, dass zelluläre Funktionen sich nicht immer auf genetische Mecha- 
nismen zurückführen lassen, sondern diese selbst Teil eines komplexen Netzwerkes sind, in dem andere biologische Faktoren und Umwelteinflüsse eine entscheidende Rolle spielen (vgl. Keller 2001; Lewontin 2001). Ein und dieselbe Genmutation kann im Hinblick auf Krankheitsverlauf und -ausprägung sehr unterschiedliche Auswirkungen haben, und umgekehrt dieselbe Krankheit von verschiedenen genetischen Veränderungen herrühren. Ungeklärt bleibt damit das zentrale Problem, wie Umwelteinflüsse und genetische Faktoren bei der Krankheitsentstehung interagieren.

\section{Wahrheitsprogramm und normative Ätiologie}

An anderer Stelle habe ich den Prozess der Genetisierung und das Paradigma einer „genetischen Medizin" als ein "Wahrheitsprogramm" bezeichnet, das eine bestimme Repräsentation von Individuum und Gesellschaft, Natur und Technologie ermöglicht (vgl. Lemke 2000). Die Verwendung dieses Begriffs zielte darauf, zwei wichtige Aspekte miteinander zu verknüpfen. Erstens ging es mir darum, den phantasmatischen Gehalt und den reduktionistischen Charakter des genetischen Determinismus herauszustellen; darüber hinaus - dies ist der zweite, entscheidende Schritt - sollte der Begriff des „Wahrheitsprogramms" deutlich machen, dass die Konzeption der DNA als Programm des Organismus, aus dem sich alle möglichen Eigenschaften und Merkmale ableiten lassen, selbst als ein Programm, eine Maschinerie zur Produktion von Wahrheit zu analysieren ist. Im Folgenden möchte ich diesen Gedanken auf der Grundlage des hier präsentierten Materials etwas konkretisieren.

Im Kontext einer molekularen Medizin von einem Wahrheitsprogramm zu sprechen, meint nicht, dass der Bezug auf genetische Faktoren im Rahmen der medizinischen Forschung einfach der materiellen Realität entbehrt. Im Gegenteil: Das Konzept der genetischen Krankheit ermöglicht eine bestimmte Konfiguration des medizinischen Wissens und erlaubt die Entwicklung neuer Interventionsformen zur Prävention, Diagnose und Therapie von Krankheiten. Die Untersuchung der „molekularen Medizin" als ein Wahrheitsprogramm macht den Einsatz einer nominalistischen Vorgehensweise notwendig, die zwei Untersuchungsdimensionen - eine archäologische und eine genealogische kombiniert. ${ }^{18}$ Zum einen ist es notwendig zu zeigen, dass genetische Erklärungen oder Krankheitsmodelle eine bestimmte grundlegende Form besitzen, die ein Set von Zeichen und Symptomen mit einer zugrunde liegenden genetischen Ursache verbindet. Dieses ätiologische Modell hat sich erst in den letzten Jahren etablieren können und blendet alternative Formen der Krankheitsanalyse aus oder drängt diese zurück. Es stellt sich also die Frage, welche $\mathrm{Zn-}$

18 Ich beziehe mit hier auf den von Michel Foucault vorgeschlagenen historisch-kritischen Nominalismus (vgl. dazu Lemke 1997: 332-339; Dean 1998; vgl. auch das Konzept eines „dynamischen" Nominalismus bei Hacking 1986). 
stände und Merkmale heute in genetische Begriffe gefasst werden, die nicht immer als genetisch bedingt oder verursacht betrachtet wurden und welche Effekte diese Form des Wissens besitzt (etwa für medizinische Praktiken, ökonomische Interessen etc.). Dies bedeutet nicht notwendig, den Geltungsanspruch des genetischen Wissens zu relativieren. ${ }^{19}$ Es geht hier also weniger um die (juridische) Frage, ob bestimmte Krankheiten zu Recht als genetische Krankheiten bezeichnet werden, sondern um die historische Untersuchung der konkreten Konstitutionsbedingungen medizinischen Wissens.

Der zweite Schritt der nominalistischen Methode baut auf dem ersten auf: Wenn bestimmte Krankheitskonzepte sich nicht mehr "von selbst verstehen", dann ist es erforderlich, das Netz von Verbindungen, Kräfteverhältnissen, Blockaden, Strategien, Zirkularitäten etc. ausfindig zu machen, das es erlaubt, zu einem gegebenen Zeitpunkt das zu etablieren, was als sozial selbstverständlich und/oder wissenschaftlich valide gilt. Die sich anschließende Frage lautet: Welche gesellschaftlichen Akteure und Interessen "profitieren ${ }^{\text {“s }}$ in welcher Form von der Genetisierung der Medizin, welche strukturellen Zwänge und institutionellen Veränderungen sind an dieses hegemoniale Modell der Krankheitsanalyse gekoppelt?

Diese Form der Analyse ermöglicht es, zwei wichtige Aspekte in den Blick zu nehmen, die in der gesellschaftlichen und wissenschaftlichen Diskussion um die Gentechnologie und deren medizinische Implikationen häufig nicht oder nur unzureichend thematisiert werden: der performative und strategische Charakter der genetischen Medizin.

Die Genetik spielt zur Zeit lediglich für medizinisch-diagnostische Anwendungen eine Rolle, in sehr viel begrenzterem Umfang in der Krankheitsprävention und bislang kaum im Bereich der Therapie. Die Gen-Medizin ist daher immer noch mehr Programm als Realität. Dennoch hat das Konzept der genetischen Krankheit entscheidend dazu beigetragen, die finanziellen und intellektuellen Ressourcen für das Humangenomprojelst bereitzustellen. Die Mobilisierung des Konzepts hat erst den Weg für jene technischen Neuerungen geschaffen, die zu Beginn des Projekts noch gar nicht existierten. ${ }^{20}$

19 Vgl. dazu Yoxen (1984: 41f.): „I take the view that the origination, development, and utilization of particular forms of knowledge, for example, about those states of conditions we call diseases, are socially determined and have to be accounted for in sociological terms. [...] One can claim, as I do, that many of the phenomena of genetic disease are grounded in a material reality while, at the same time, asking why we isolate or delineate certain phenomena for analysis, why we say that they constitute diseases, and why we seek to explain their nature and cause in genetic terms. [...] In seeking to consider the sociology of genetic explanations of disease, we are essentially asking what kinds of evidence and explanation persuade."

20 Vgl. Rheinberger (1996: 293): „In den Anfängen des Genomprojekts stand keines der Mittel zur Verfügung, nit dem die Aufgabe einer Sequenzierung der ca. drei Milliarden Basenpaare eines menschlichen Genoms mit Aussicht auf Erfolg auch nur hätte in Angriff genonmen werden können. Das Programm selbst hat einen Mechanismus in Gang gesetzt, über den diese Mittel überhaupt erst Gestalt annehmen." 
Zugleich war die mediale Berichterstattung und die Popularisierung des Ansatzes einer Gen-Medizin eine entscheidende Voraussetzung für deren gesellschaftliche Akzeptanz:

„Der Begriff der genetischen Krankheit, den sich die medizinischen Wissenschaften aus komplexen institutionellen und wirtschaftlichen Gründen zu eigen gemacht haben, spiegelt eine ideologische Expansion der Molekularbiologie wider, die weit über ihre technischen Erfolge hinausreicht. Außerdem hat die allgemeine Akzeptanz dieser Vorstellung ihrerseits entscheidend dazu beigetragen, den Weg für die nachfolgenden technischen Entwicklungen in der Molekularbiologie zu ebnen." (Keller 1995: 297)

Neben dieser performativen Dimension ist die strategische Ausrichtung des Gendiskurses zu untersuchen. Hierbei sind zunächst die veränderten strukturellen Zwänge und institutionellen Selektionsmechanismen innerhalb des Gesundheitssystems zu berücksichtigen:

„Health-related conditions have to be packaged so as to fit the institutional, professional, and conceptual structure of modern medicine. In the case of conditions (rightly or wrongly) to be genetic in cause, the mechanistic basic explanatory form of a genetic disease' has been constructed to fit the contemporary context. This is not to say that our understanding of particular conditions is necessarily invalid, misleading, or the result of deliberate misrepresentation, but it is to say that medical conceptualization of particular conditions bear the mark of a struggle over whether and how to think about them." (Yoxen 1984: 48)

Der Aufstieg der Genetik innerhalb der Medizin und das Aufkommen des Konzepts einer genetischen Krankheit ist jedoch nicht allein auf interne Strukturen des medizinischen Komplexes oder professionspolitische Profilierungsstrategien zurückzuführen, sondern auch im Kontext allgemeinerer gesellschaftlicher Veränderungen zu analysieren. ${ }^{21}$ Was als Krankheit anzusehen und wie diese zu behandeln sein soll, ist Gegenstand von sozialen Konflikten und politischen Auseinandersetzungen. Die Konzentration auf genetische Krankheitsursachen (und die gleichzeitige Vernachlässigung anderer Krankheitsfaktoren) ist also mehr als ein innerwissenschaftliches Phänomen oder ein Resultat medizinisch-technischen Fortschritts. Die Karriere bestimmter Krankheitsdeutungen hängt nicht zuletzt davon $\mathrm{ab}$, ob sie in allgemeinere gesundheits- und gesellschaftspolitische Strategien "passen“. Ob konkrete Gesundheits- bzw. Krankheitskonzepte politische Berücksichtigung finden, als sozial akzeptabel und wissenschaftlich respektabel erscheinen, ist von gesellschaftlichen Selektionsmechanismen ebenso wie von politischen Interessen abhängig. Hagen Kühn hat daher von einer „normativen Ätiologie“ gesprochen. Ihm zufolge zeigt

„die Empirie des praktischen Interventionsgeschehens und der unmittelbar auf die Praxis zielenden Forschung und ihrer Förderung Regelmäßigkeiten einer gesellschaftlichen ,Zuchtwahl‘ von ä-

21 Eine entscheidende Rolle spielt dabei auch die Bildung von Allianzen und strategischen Koalitionen, die neue Einfluss-, Verwertungs- und Gestaltungschancen für die beteligten Akteure eröffnen: „Die Molekularbiologie verbündet sich mit dem humangenetischen Beratungssystem, dem medizinisch-technischen Komplex, der Biotechnologie-Industrie und der forensischen Medizin, und indem sie dies tut, werden sich alle verändern, und ein neues medizinisches Paradigma wird entstehen: die molekulare Medizin.“ (Rheinberger 1996: 293; 292-294) 
tiologischen Konzepten. [...] „Survival of the fittest ${ }^{\varsigma}$ meint dabei nicht die Auswahl des Stärksten oder des Zutreffendsten, sondern des am besten am Status quo Angepaßten [... Ergebnis dieser gesellschaftlichen, d. h. interessen- und machtbedingten "Zuchtwahl ${ }^{\prime}$ sind nicht analytische, sondern letztlich normative Ätiologien, die sich freilich - da Wissenschaftsförmigkeit Legitimationsbedingung für Gesundheitskonzepte ist - als solche nicht zu erkennen geben dürfen." (Kühn 2000: 13; vgl. auch Tesh 1988)

Der implizit normative „ätiologische Diskurs ${ }^{\text {ss }}$ enthalte sowohl die geläufigen Alltagsvorstellungen als auch die jeweils hegemonialen wissenschaftlichen Positionen zu den Krankheitsursachen. Kühns These ist, dass die „Überlebensbedingungen ${ }^{\text {"s }}$ und Durchsetzungskraft ätiologischer Konzepte entscheidend von politischen Kräfteverhältnissen und Interessenkonstellationen sowie den praktischen Handlungskonsequenzen abhängen. Ohne die Existenz wissenschaftlich gesicherter Krankheitsbedingungen in Frage zu stellen, macht er plausibel, dass einige Krankheitskonzepte und Interventionsmodelle innerhalb der gesellschaftlichen Verhältnisse eine größere "Chance" besitzen, wissenschaftlich thematisiert und zum Gegenstand praktischen gesundheitsbezogenen Handelns zu werden. Diese Berücksichtigungschancen gehen nicht auf deren innere Konsistenz oder wissenschaftliche Exzellenz zurück, sondern sind eher ihrer „Korrespondenz" mit außerwissenschaftlichen strukturellen Zwängen geschuldet. In dieser Perspektive haben Krankheitskonzepte nicht nur mit wissenschaftlichen Irrtümern und Erkenntnisbarrieren zu kämpfen, sondern auch und vor allem mit Macht- und Verteilungsinteressen.

Von dieser Annahme ausgehend unterscheidet Kühn im Rahmen eines Evolutionsmodells verschiedene "Stufen" von Krankheitskonzepten nach der ihnen eigenen „Überlebenswahrscheinlichkeit“. Die besten Durchsetzungschancen räumt er Konzepten ein, die sich auf isolierbare Krankheitserreger und genetische Bedingungen konzentrieren, da sich die Krankheitsursachen in diesem Fall allein im Bereich der Mikroereignisse des individuellen Körpers lokalisieren lassen - und damit zugleich der umgreifendere soziale Kontext unberührt bleibt. Daher dürfte die Genetisierung des Krankheitsverständnisses

„zu einem großen Teil auf die Verheißung zurückzuführen sein, hier könne Krankheit bekämpft werden, ohne den sozialen und politischen Status quo zu verletzen. Mehr noch; dieser wird gestärkt durch marktförmige Befriedigung von Gesundheitsbedürfnissen, durch neue Märkte für Kapitalanleger und durch den Legitimationsentzug für Interventionen in den sozialen Kontext von Gesundheit und Krankheit. [...] Auf der zweiten Stufe der ätiologischen Hierarchie stehen jene Krankheitsursachen, die unmittelbar als ein reflexives, d. h. auf sich selbst bezogenes, Fehlverhalten' der Individuen angesehen werden, also die subjektiven Risikofaktoren des sogenannten Lebensstils (Mangel an körperlicher Bewegung, falscher und übermäßiger Ernährung, zuviel Alkohol und Tabak, falsche Sozialbeziehungen)" (Kühn 2000: 14 f.; Hervorhebung im Orig.).

Von diesen privilegierten Krankheitsursachen grenzt Kühn weitere Stufen ätiologischer Diskurse ab, deren gesellschaftliche Berücksichtigung wesentlich schwieriger sei. Hierzu gehören Faktoren der physischen Umwelt (wie etwa fehlende Arbeitssicherheit) ebenso wie übergreifende gesellschaftliche Verhältnisse. Hier zu intervenieren sei besonders schwierig: 
„Nirgendwo ist das Geld knapper, sind die Legitimationskosten höher und ist die Beweislast schwerer als bei Versuchen, in Bereiche wie Arbeit, Verkehr oder Wohnbedingungen oder soziale Probleme wie Armut, Erwerbs- oder Machtlosigkeit unter Gesundheitsaspekten zu intervenieren " (Kühn 2000: 16).

In dieser Hinsicht erfüllt ein genetisiertes Krankheitsverständnis zwei wichtige Funktionen, die entscheidend zur gesellschaftlichen Etablierung der GenMedizin beitragen dürften. Erstens ermöglicht sie die Lokalisierung pathogener Faktoren im Individuum selbst, ohne den umfassenden sozialen und physischen Kontext der Pathogenese systematisch einzubeziehen. Zweitens erlaubt das Modell genetischer Krankheiten die Vision einer „individualisierten", d.h. auf das individuelle Genom abgestimmten Medizin, zu verfolgen. Die GenMedizin verspricht also eine "Bewältigung" von Krankheitsproblemen, die es nicht nur scheinbar überflüssig macht, pathogene gesellschaftliche Verhältnisse zu ändern, sondern bietet auch marktförmige "Problemlösungen " in Form von neuen Medikamenten, Diagnostika und Präventionsoptionen an. ${ }^{22}$

So überzeugend das von Kühn vorgeschlagene Analysemodell insgesamt ist, an einem wichtigen Punkt ist eine Korrektur notwendig. Die wissenschaftliche Dominanz und die gesellschaftliche Akzeptanz der gegenwärtigen Genetisierung von Krankheitsursachen beruht weniger auf einer hierarchischen Stufung als auf einer strategischen Kopplung von erster und zweiter Ebene, die über das Konzept des (genetischen) Risikos funktioniert. ${ }^{23}$ Anders als Kühn annimmt, sind Gene nicht nur im Individuum als potentielle Krankheitsursachen zu lokalisieren, vielmehr soll es möglich sein, genetische Risiken ähnlich wie andere Gesundheitsrisiken durch entsprechende Handlungsoptionen zu minimieren. ${ }^{24}$ In dieser Hinsicht stellt das genetische Wissen Informationen bereit, welche die Grundlage für ein "rationales" Gesundheitsverhalten der Individuen bilden. In Zukunft könnten genetische Bedingungen zunehmend wie subjektive Risikofaktoren behandelt werden und sich neue individuelle Entscheidungszwänge und moralische Verpflichtungen etablieren. Vor allem in den USA zeichnen sich bereits heute Konturen einer „genetischen Verantwortung“ ab, die neoliberale Forderungen nach einer stärkeren gesellschaftlichen

22 Ein interessantes Illustrationsbeispiel für eine "normative Ätiologie" und die Abhängigkeit der Krankheitskonzepte von politischen Konjunkturen bietet Nicola Wolfs Analyse der Transformation des Krebsverständnisses in den USA von den 1970er Jahren bis heute. Konzentrierte sich das medizinische Interesse in der innenpolitischen Reformperiode der 1970er Jahre auf die Untersuchung von industriell erzeugten Umweltschadstoffen, wurde in der Reagan-Ära die Rolle von individuellen Risikofaktoren wie Tabakkonsum oder Alkohol in den Vordergrund gestellt; in den 1990er Jahren kam es dann schließlich zur Hegemonie der Genetik in der Krebsforschung (Wolf 2000; ausführlich: Proctor 1995).

23 Vgl. dazu ausführlicher Lemke (2000; 2001; 2002).

24 Ein weiterer Kritikpunkt: Das von Kühn vorgeschlagene Analysemodell ist zu statisch konzipiert, da im Zentrum die "Anpassung "von Krankheitskonzepten an einen bereits existierenden gesellschaftlichen Status quo steht. Die Folge ist, dass der Aspekt der Performanz, also die Frage, inwieweit Diskurse und Praktiken den Status quo selbst verändern bzw. ihn konstituieren (statt ihm äußerlich gegenüber zu stehen), nicht in den Blick kommt. 
Akzentuierung von Eigenverantwortung und Selbstsorge aufnimmt und reproduziert:

"This shift in responsibility from society to the individual aligns with our current political climate, which increasingly blames individuals rather than social conditions for human problems. Thus genetics could become part of an ideological shift away from environmental and social analyses of problems, fostering the decline of public responsibility for human misfortune and misery." (Conrad 2002: 76; Bause 2000; Petersen/Bunton 2002). ${ }^{25}$

Festzuhalten ist, dass das Konzept des genetischen Risikos es erlaubt, den Krankheitsdiskurs mit der Vorstellung von medizinischer Steuerbarkeit und individueller Zurechnung zu verbinden. Im Zentrum der molekularen Medizin steht daher nicht die Feststellung einer faktischen Determinationsbeziehung, sondern die Eröffnung von praktischen Interventionsmöglichkeiten und der Einsatz von Techniken moralischer Regulation. Innerhalb der gegenwärtigen gesellschaftlichen Entwicklungstrends könnte die Gen-Medizin nicht nur die Tendenz verstärken, Krankheitsursachen im individuellen Körper zu lokalisieren; darüber hinaus besteht die Gefahr, dass Verantwortung und Kosten für Krankheiten vermehrt den direkt Betroffenen subjektiv zugerechnet werden.

\section{Literatur}

Bause, Margarete (2000): Guter Rat ist teuer - humangenetische Beratung unter den Bedingungen der Marktindividualisierung, in: Schmidtke, Jörg ( $\mathrm{Hg}$.), Guter Rat ist teuer. Was kostet die Humangenetik, was nutzt sie?, München/Jena: Urban \& Fischer, 96-106.

Bell, John (1998): The new genetics: The new genetics in clinical practice, British Medical Journal, Vol. 316, No. 7131, 618-620.

Brandt, Allan M./Rozin, Paul (1997): Morality and Health, New York/London: Routledge.

Bulmahn, Edelgard (2003): Vorwort, in: Bundesministerium für Bildung und Forschung ( $\mathrm{Hg}$.) Krankheitsbekämpfung durch Genomforschung. Das Nationale Genomforschungsnetz, Bonn: $\mathrm{BMBF}, 6-7$.

Carson, Ronald A./Rothstein, Mark A. (1999): Behavioral Genetics. The Clash of Culture and Biology, Baltimore/London: The John Hopkins University Press.

Caskey, C. Thomas (1995): Medizin auf der Grundlage der DNA - Prävention und Therapie, in: Kevles, Daniel J./ Hood, Leroy (Hg.), Der Supercode. Die genetische Karte des Menschen, Frankfurt am Main: Insel, 123-155.

Chadarevian, Soraya de/Kamminga, Harmke (Hg.) (1998): Molecularizing Biology and Medicine. New Practices and Alliances, 1910s-1970s, Amsterdam: Overseas Publishers Association.

Childs, Barton (1977): Persistent Echoes of the Nature-Nurture Argument, American Journal of Human Genetics, Vol. 29, 1-13.

Childs, Barton (1999): Genetic Medicine. A Logic of Disease, Baltimore/London: The John Hopkins University Press.

Clark, William R. (1997): The New Healers. The Promise and Problems of Molecular Medicine in the Twenty-First Century, New York/Oxford: Oxford University Press.

25 Die Konturen einer „genetischen Verantwortung" zeigt etwa B. Irrgang sehr deutlich auf: „Patienten im Sinne der prädiktiven Medizin sind daher keine Kranken im eigentlichen Sinn, sondern Menschen, die Aufklärung über genetische Informationen haben wollen, die medizinische Relevanz haben kann. Potentiell Kranke erhalten damit eine neue Verantwortung für ihre zukünftige Erkrankung, insbesondere dann, wenn Kinder oder andere Familienangehörige ebenfalls aktuell oder potentiell von einem Erkrankungsrisiko betroffen sein könnten" (2001: 652; vgl. auch Sass 1994: 344 f.). 
Conrad, Peter (1999): A Mirage of Genes, Sociology of Health \& Illness, Vol. 21, No. 2, 228-241.

Conrad, Peter (2002): Genetics and Behavior in the News: Dilemmas of a Rising Paradigm, in: Alper, Joseph S. et al. (Hg.), The Double-Edged Helix. Social Implications of Genetics in a Diverse Society, Baltimore/London: The John Hopkins University Press, 58-79.

Dean, Mitchell (1998): Questions of Method, in: Velody, Irving/Williams, Robin (Hg.), The Politics of Constructionism, London/Thousand Oaks/New Dehli: Sage, 182-199.

Emery, Jon/Hayflick, Susan (2001): The challenge of integrating genetic medicine into primary care, British Medical Journal, Vol. 322, 1027-1030.

Fadiman, Anne (2000): Der Geist packt dich und du stürzt zu Boden, Berlin: Berlin Verlag.

Freidson, Eliot (1979): Der Ärztestand: Berufs- und wissenschaftssoziologische Durchleuchtung einer Profession, Stuttgart.

Ganten, Detlev/Ruckpaul, Klaus (1997ff): Handbuch der Molekularen Medizin, 12 Bände, Heidelberg u. a.: Springer.

Ganten, Detlev/Ruckpaul, Klaus (2001): Molekulare Medizin, in: Raem, A. M. et al., GenMedizin. Eine Bestandsaufnahme, Berlin u. a.: Springer, 3-19.

Hacking, Ian (1986): Making Up People, in: Heller, Thomas/Sosna, Morton/Wellberry, David E. (Hg.), Reconstructing Individualism. Autonomy, Individuality, and the Self in Western Thought, Stanford: Stanford University Press, 222-236.

Harris, Rodney/Harris, Hilary J (1995): Primary Care for Patients at Genetic Risk, British Medical Journal, Vol. 311, 579-80.

Hoedemaekers, Rogeer Hubertus Maria Vincent (1998): Normative Determinants of Genetic Screening and Testing, Wageningen.

Hoedemaekers, Rogeer/Ten Have, H. (1999): The Concept of Abnormality in Medical Genetics, Theoretical Medicine and Bioethics, Vol, 20, No. 6, 537-561.

Höhn, Holger (1997): Die Genetifizierung der Medizin und die Zukunft der Humangenetik, Medizinische Genetik, 9. Jg., 173-174.

Irrgang, B. (2001): Der Krankheitsbegriff der prädiktiven Medizin und die humangenetische Beratung, in: Raem, Arnold Maria et al, Gen-Medizin. Eine Bestandsaufnahme, Berlin u. a.: Springer, $651-660$.

Janich, Peter (2001): Der Status des genetischen Wissens, in: Honnefelder, Ludger/Propping, Peter ( $\mathrm{Hg}$.), Was wissen wir, wenn wir das menschliche Genom kennen?, Köln: Dumont, 70-89.

Kaplan, J. C./Junien, C. (2000): Genomics and Medicine: an Anticipation. From Boolean Mendelian Genetics to Multifactorial Molecular Medicine, Compres Rendus De L'Academie Des Sciences, Serie lir-Sciences De La Vie-Life Sciences, Vol. 323, No. 12, 1167-1174.

Kaprio, J. (2001): "Commentary: Role of Other Genes and Environment Should Not Be Overlooked in Monogenic Disease", British Medical Journal, Vol. 322, 1023.

Keller, Evelyn Fox (1995): Erbanlage, Umwelt und das Genomprojekt, in: Kevles, D. J./Hood, L. (Hg.), Der Supercode. Die genetische Karte des Menschen, Frankfurt am Main/Leipzig: Insel, S. 284-303.

Keller, Evelyn Fox (2001): Das Jahrhundert des Gens, Frankfurt am Main: Campus.

Kerr, Anne (2000): (Re)Constructing Genetic Disease: The Clinical Continuum between Cystic Fybrosis and Male Infertality, Social Studies of Science, Vol. 30, No. 6, 847-894.

Kitcher, Philip (1998): Genetik und Ethik. Die Revolution der Humangenetik und ihre Folgen, München: Luchterhand.

Koch, Lene (1993): The Genefication of Medicine and the Concept of Disease, in: Hamburger Institut für Sozialforschung $(\mathrm{Hg}$.) Diskussionpapiere 1/93, Hamburg: Hamburger Institut für Sozialforschung.

Koch, Lene (1999): Predictive genetic medicine - a new concept of disease, in: Hildt, Elisabeth and Graumann, Sigrid (Hg.), Genetics in Human Reproduction, Aldershot u. a.: Ashgate, 185-195.

Kühn, Hagen (2000): Normative Ätiologie. Zur Herrschaftlichkeit des gesellschaftlichen Krankheitsverständnisses, Jahrbuch für Kritische Medizin, Nr. 34, 11-18.

Labisch, Alfons (2002): Gesundheit und Medizin im molekularen Zeitalter - Eine historische Vorausschau, in: Honnefelder, Ludger/Propping, Peter (Hg.), Was wissen wir, wenn wir das menschliche Genom kennen??, Köln: Dumont, 128-136.

Latour, Bruno (1988): The Pasteurization of France, Cambridge: Cambridge University Press.

Lau, Christoph/Keller, Reiner (2001): Zur Politisierung gesellschaftlicher Naturabgrenzungen, in: 
Beck, Ulrich/BonB, Wolfgang (Hg.), Die Modernisierung der Moderne, Frankfurt am Main: Suhrkamp, 82-95.

Lemke, Thomas (1997): Eine Kritik der politischen Vernunft. Foucaults Analyse der modernen Gouvernementalität. Hamburg: Argument.

Lemke, Thomas (2000): Die Regierung der Risiken. Von der Eugenik zur genetischen Gouvernementalität, in: Bröckling, Ulrich/Krasmann, Susanne/Lemke, Thomas (Hg.), Gouvernementalität der Gegenwart. Studien zur Ökonomisierung des Sozialen, Frankfurt am Main: Suhrkamp.

Lemke, Thomas (2001): Zurück in die Zukunft? Genetische Diagnostik und das Risiko der Eugenik. in: Graumann, Sigrid (Hg.), Die Genkontroverse, Freiburg im Br: Herder, 37-44.

Lemke, Thomas (2002): Mutationen des Gendiskurses. Der genetische Determinismus nach dem Humangenomprojekt, Leviathan, 30. Jg., Heft 3, 400-425.

Lewontin, Richard (2001): The Triple Helix. Gene, Organism and Environment, Cambridge, MA/London: Harvard University Press.

Marteau, Theresa M./Neil A. Holtzman. (2000): Will Genetics Revolutionize Medicine? New England Journal of Medicine 343 (2), 141-44.

Maurer, Johannes/Lehrach, Hans (2001): Geleitwort, in: Raem, A. M. et al., Gen-Medizin. Eine Bestandsaufnahme, Berlin u. a.: Springer, XIII-XIV.

Morris, David B. (2000): Krankheit und Kultur. Plädoyer für ein neues Körperverständnis, München: Antje Kunstmann.

Paul, Diane (1998a): A Debate That Refuses To Die, in: Paul, Diane, The Politics of Heredity. Essays on Eugenics, Biomedicine, and the Nature-Nurture Debate, Albany: State University of New York Press, 81-93.

Paul, Diane (1998b): PKU Screening: Competing Agendas, Converging Stories, in: Paul, Diane, The Politics of Heredity. Essays on Eugenics, Biomedicine, and the Nature-Nurture Debate, Albany: State University of New York, 173-186.

Petersen, Alan/Bunton, Robin (2002): The new genetics and the public's health, London/New York: Routledge.

Porter, Roy (1997): The Greatest Benefit to Mankind. A Medical History of Humanity from Antiquity to the Present, London: HarperCollins.

Proctor, Robert N. (1995): Cancer Wars. How Politics Shapes What We Know And Don't Know About Cancer, New York: Basis Books.

Raem, Arnold Maria et al. (2001): Gen-Medizin. Eine Bestandsaufnahme, Berlin/Heidelberg u. a.: Springer.

Rapp, Rayna (2000): Testing Women, Testing the Fetus. The Social Impact of Amniocentesis in America, New York/London: Routledge.

Rees, Jonathan (2002): Complex Disease and the New Clinical Sciences, Science, Vol. 296, 698-701.

Rheinberger, Hans-Jörg (1996): Jenseits von Natur und Kultur. Anmerkungen zur Medizin im Zeitalter der Molekularbiologie. in: Borck, Cornelius (Hg.), Anatomien medizinischen Wissens, Frankfurt am Main: Fischer, 287-306.

Rheinberger, Hans-Jörg (1997): Von der Zelle zum Gen. Repräsentationen der Molekularbiologie, in: Rheinberger, Hans-Jörg/Hagner, Michael/Wahrig-Schmidt, Bettina (Hg.), Räume des Wissens, Repräsentation, Codierung, Spur, Berlin: Akademie Verlag, 265-279.

Rheinberger, Jörg (1998): Bemerkungen zur Geschichte der Molekularbiologie, in: Kunst- und Ausstellungshalle der Bundesrepublik Deutschland ( $\mathrm{Hg}$.), Gen-Welten, Köln: Dumont, 58-64.

Rosenstock, IM et al. (1975): Genetic Screening. A Study of the Knowledge and Attitudes of Physicians, Washington D.C.: National Academy of Sciences.

Rushton, Alan R. (1994): Genetics and Medicine in the United States 1800-1922, Baltimore/London: The John Hopkins University Press.

Sass, Hans-Martin (1994): Der Mensch im Zeitalter von genetischer Diagnostik und Manipulation. Kultur, Wissen und Verantwortung, in: Fischer, Ernst Peter/Geißler, Erhard (Hg.), Wieviel Genetik braucht der Mensch? Die alten Träume der Genetiker und ihre heutigen Methoden, Konstanz: Universitätsverlag Konstanz, 339-353.

Schmidtke, Jörg (1998): Die Genetisierung der Medizin, Public Health Forum, Nr. 19, 12.

Smith, Kelly C. (2001): A disease by any other name: Musings on the concept of genetic disease, Medicine, Health Care and Philosophy, Vol. 4, 19-30.

Strachan, Tom/Read, Andrew P. (1996): Molekulare Humangenetik, Heidelberg u.a.: Spektrum Akademischer Verlag. 
Temple, Larissa K F. et al. (2001): Defining disease in the genomics era, Science, Vol. 293, 807-808.

Tesh, Sylvia Noble (1998): Hidden Arguments. Political Ideology and Disease Prevention Policy, New Brunswick/London: Rutgers University Press.

Van den Boer-van den Berg/Hanneke M. A./Maat-Kievit, Anneke A. (2001): The whole truth and nothing but the truth, but what is the truth?, Journal of Medical Genetics, Vol. 38, 39-42.

Wailoo, Keith (2001): Dying in the City of the Blues. Sickle Cell Anemia and the Politics of Race and Health, Chapel Hill und London: The University of Northern Carolina.

Wasserman, David/Wachbroit, Robert (2001): Genetics and Criminal Behavior, Cambridge: Cambridge University Press.

Wiesing, Urban (1998): Gene, Krankheit und Moral, in: Kunst- und Ausstellungshalle der Bundesrepublik Deutschland (Hg.), Gen-Welten, Köln: Dumont, 95-99.

Willett, Walter C. (2002): Balancing Life-Style and Genomics Research for Disease Prevention. Science, Vol. 296, 695-698.

Williams, S. J./Hayward, N. K (2001): The Impact of the Human Genome Project on Medical Genetics, Trends in Molecular Medicine, May; 7 (5), 229-231.

Wolf, Nicola (2000): Genetische Hoffnungen. Zum Wandel des Krankheitsverständnisses bei Krebs, Jahrbuch fü Kritische Medizin, Nr. 34, 61-81.

Yoxen, Edward J. (1984): Constructing Genetic Diseases, in: Duster, Troy/Garrett, Karen (Hg.), Cultural Perspectives on Biological Knowledge, Norwood/NJ: Ablex Publishing Corporation, 41-62.

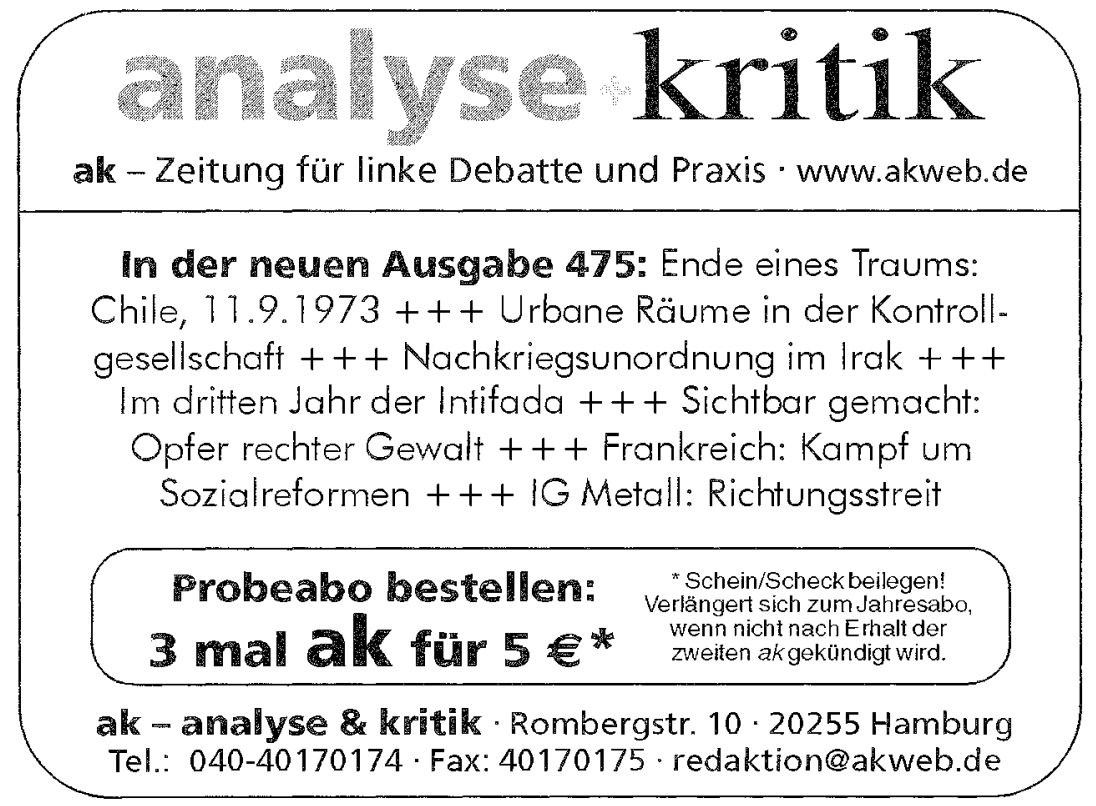

